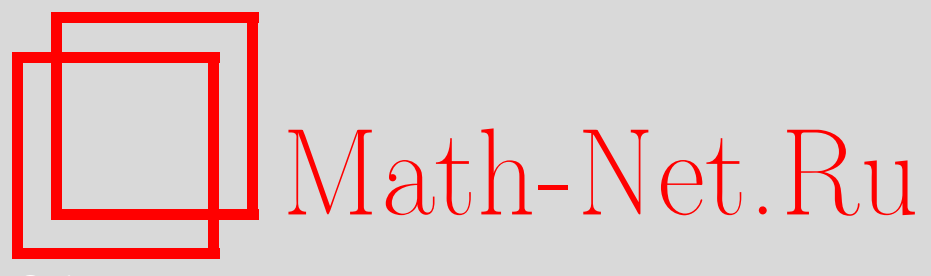

С. Я. Новиков, Бесселевы последовательности как проекции ортогональных систем, Матем. заметки, 2007, том 81, выпуск 6, 893-903

DOI: https://doi.org/10.4213/mzm3739

Использование Общероссийского математического портала Math-Net.Ru подразумевает, что вы прочитали и согласны с пользовательским соглашением http://www . mathnet.ru/rus/agreement

Параметры загрузки:

IP: 54.210 .77 .194

26 апреля 2023 г., $07: 51: 59$

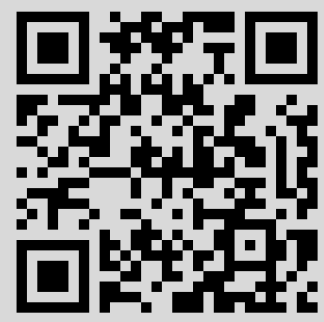


Том 81 выпуск 6 июнь 2007

УДК $517.51+517.98$

\section{Бесселевы последовательности как проекции ортогональных систем}

\section{С. Я. Новиков}

Доказаны обобщения теорем Шура и Олевского о продолжении систем функций с интервала $I$ до ортогональных систем на интервале $J, I \subset J$. Бесселевы системы в $L^{2}(I)$ и только они являются проекциями ортогональных систем из более широкого пространства $L^{2}(J)$. Этот факт позволяет одним методом перенести классические теоремы о сходимости почти всюду ортогональных рядов (Меньшова-Радемахера, Пэли-Зигмунда, Гарсиа) на ряды по бесселевым системам. Проекция полной ортогональной системы из $L^{2}(J)$ на $L^{2}(I)$ является жестким фреймом, но не базисом.

Библиография: 10 названий.

Основными объектами работы являются два вида последовательностей в комплексном гильбертовом пространстве - бесселевы последовательности и фреймы (каркасы). Они стали весьма популярными в математической литературе последних лет, как теоретической, так и прикладной направленности [1]-[4].

ОПредЕЛЕНиЕ 1. Последовательность $\mathscr{F}=\left\{f_{k}\right\}_{k=1}^{\infty}$ элементов гильбертова пространства $\mathscr{H}$ называется бесселевой, если существует константа $B>0$ такая, что

$$
\sum_{k=1}^{\infty}\left|\left\langle f, f_{k}\right\rangle\right|^{2} \leqslant B\|f\|^{2}, \quad f \in \mathscr{H} .
$$

Число $B$ называется границей бесселевой последовательности. Точную нижнюю грань всех возможных границ бесселевой последовательности называют оптимальной границей бесселевой последовательности.

Лемма 2. Ортогональная система $\left\{\varphi_{k}\right\}_{k=1}^{\infty}$ такая, что $\left\|\varphi_{k}\right\|=\sqrt{B}, k=1,2, \ldots$, является бесселевой последовательностъю с границей $B$.

ДокАЗАтЕЛЬСтво. Для ортонормированной системы $f_{k}=\varphi_{k} / \sqrt{B}$ имеет место классическое неравенство Бесселя

$$
\sum_{k=1}^{\infty}\left|\left\langle f, f_{k}\right\rangle\right|^{2} \leqslant\|f\|^{2}, \quad f \in \mathscr{H},
$$

из которого следует, что

$$
\sum_{k=1}^{\infty}\left|\left\langle f, \varphi_{k}\right\rangle\right|^{2} \leqslant B\|f\|^{2}, \quad f \in \mathscr{H} .
$$

(C) С. Я. Новиков, 2007 
С бесселевой последовательностью связаны два оператора: оператор

$$
C=C_{\mathscr{F}}: \mathscr{H} \rightarrow \ell^{2}, \quad C_{\mathscr{F}} f=\left\{\left\langle f, f_{k}\right\rangle\right\}_{k=1}^{\infty}
$$

называется оператором анализа, оператор

$$
D=D_{\mathscr{F}}: \ell^{2} \rightarrow \mathscr{H}, \quad D_{\mathscr{F}}\left\{c_{k}\right\}_{k=1}^{\infty}=\sum_{k=1}^{\infty} c_{k} f_{k}
$$

- оператором синтеза. Операторы $C$ и $D$ являются взаимно сопряженными.

Лемма 3. Последовательность $\mathscr{F}=\left\{f_{k}\right\}_{k=1}^{\infty}$ является бесселевой в $\mathscr{H}$ с граниией $B$ тогда и толъко тогда, когда оператор синтеза $D_{\mathscr{F}}$ ограничен из $\ell^{2}$ в $\mathscr{H} u$ $\|D\| \leqslant \sqrt{B}$.

ДокАЗАТЕЛЬСтво. Пусть $\left\{f_{k}\right\}_{k=1}^{\infty}-$ бесселева последовательность с границей $B$ и $\left\{c_{k}\right\}_{k=1}^{\infty} \in \ell^{2}$. Покажем, что ряд $\sum_{k=1}^{\infty} c_{k} f_{k}$ сходится. Пусть $n, m \in \mathbb{N}$ и $n>m$. Тогда

$$
\begin{aligned}
\left\|\sum_{k=1}^{n} c_{k} f_{k}-\sum_{k=1}^{m} c_{k} f_{k}\right\| & =\left\|\sum_{k=m+1}^{n} c_{k} f_{k}\right\|=\sup _{\|g\|=1}\left|\left\langle\sum_{k=m+1}^{n} c_{k} f_{k}, g\right\rangle\right| \\
& \leqslant \sup _{\|g\|=1} \sum_{k=m+1}^{n}\left|c_{k}\left\langle f_{k}, g\right\rangle\right| \\
& \leqslant\left(\sum_{k=m+1}^{n}\left|c_{k}\right|^{2}\right)^{1 / 2} \sup _{\|g\|=1}\left(\sum_{k=m+1}^{n}\left|\left\langle f_{k}, g\right\rangle\right|^{2}\right)^{1 / 2} \\
& \leqslant \sqrt{B}\left(\sum_{k=m+1}^{n}\left|c_{k}\right|^{2}\right)^{1 / 2} .
\end{aligned}
$$

Так как $\left\{c_{k}\right\}_{k=1}^{\infty} \in \ell^{2}$, из проведенных преобразований получаем, что ряд $\sum_{k=1}^{\infty} c_{k} f_{k}$ сходится и $\|D\| \leqslant \sqrt{B}$.

Для доказательства обратной импликации предположим, что оператор синтеза ограничен и $\|D\| \leqslant \sqrt{B}$. Тогда сопряженный оператор, оператор анализа $C$, также ограничен и мы приходим к определению бесселевой последовательности.

Заметим, что определение бесселевой последовательности не зависит от порядка следования элементов последовательности. Это простое замечание приводит к важному следствию.

СледствиЕ 4. Если $\left\{f_{k}\right\}_{k=1}^{\infty}-$ бесселева последовательность в $\mathscr{H}$, то ряд

$$
\sum_{k=1}^{\infty} c_{k} f_{k}
$$

сходится безусловно для всех $\left\{c_{k}\right\}_{k=1}^{\infty} \in \ell^{2}$.

Для бесселевой последовательности $\mathscr{F}=\left\{f_{k}\right\}_{k=1}^{\infty}$ определен ограниченный оператор

$$
C \circ D: \ell^{2} \rightarrow \ell^{2}, \quad C \circ D\left\{c_{k}\right\}_{k=1}^{\infty}=\left\{\left\langle\sum_{l=1}^{\infty} c_{l} f_{l}, f_{k}\right\rangle\right\}_{k=1}^{\infty} .
$$


Матричное представление этого оператора в стандартном базисе $\left\{e_{k}\right\}_{k=1}^{\infty}$ ортов пространства $\ell^{2}$ имеет вид

$$
(C \circ D)_{j, k}=\left\langle C \circ D e_{k}, e_{j}\right\rangle=\left\langle D e_{k}, D e_{j}\right\rangle=\left\langle f_{k}, f_{j}\right\rangle,
$$

т.е. является матрицей Грама $G_{\mathscr{F}}$ последовательности $\mathscr{F}$. Известно [4; п. 3.5.1], что матрица Грама $G_{\mathscr{F}}$ определяет ограниченный на $\ell^{2}$ оператор с $\left\|G_{\mathscr{F}}\right\| \leqslant B$ в том и только том случае, когда последовательность $\mathscr{F}$ бесселева с границей $B$. Нам понадобится уточнение последнего результата.

Лемма 5. Последовательность Я્F бесселева с границей $B$ в том и только том случае, когда ее матрица Грама удовлетворяет неравенствам $0 \leqslant G_{\mathscr{F}} \leqslant B \mathbf{I}$, где $\mathbf{I}$ - тождественный оператор пространства $\ell^{2}$.

ДокАЗАТЕЛьство. Если последовательность бесселева, то

$$
\left\langle G_{\mathscr{F}} c, c\right\rangle=\left\langle C_{\mathscr{F}} \circ D_{\mathscr{F}} c, c\right\rangle=\left\langle D_{\mathscr{F}}^{*} \circ D_{\mathscr{F}} c, c\right\rangle=\left\langle D_{\mathscr{F}} c, D_{\mathscr{F}} c\right\rangle \geqslant 0, \quad c \in \ell^{2},
$$

и в силу леммы 3

$$
\left\langle G_{\mathscr{F}} c, c\right\rangle=\left\|\sum_{k=1}^{\infty} c_{k} f_{k}\right\|^{2} \leqslant B\|c\|^{2}=B\langle c, c\rangle, \quad c \in \ell^{2} .
$$

С другой стороны, из неравенства $B \mathbf{I}-G_{\mathscr{F}} \geqslant 0$ получаем

$$
\left\|\sum_{k=1}^{\infty} c_{k} f_{k}\right\|^{2} \leqslant B\|c\|^{2}, \quad c \in \ell^{2}
$$

что эквивалентно (лемма 3 ) бесселевости последовательности $\mathscr{F}$.

Лемма 6. Если матрица А определяет ограниченный неотрицательный оператор в пространстве $\ell^{2}$, то в гильбертовом пространстве $\mathscr{H}$ существует последовательность элементов $\mathscr{F}=\left\{f_{k}\right\}_{k=1}^{\infty}$ такая, что матрица Грама $G_{\mathscr{F}}=A$.

ДокАЗАтЕЛьство. В комплексном гильбертовом пространстве каждый ограниченный неотрицательный оператор $A$ является самосопряженным. Для него однозначно определен неотрицательный (следовательно, самосопряженный) оператор $\sqrt{A}$ такой, что $(\sqrt{A})^{2}=A\left[5 ;\right.$ п. 12.33]. В пространстве $\ell^{2}$ последовательность $\mathscr{F}$ образована строками матрицы, которая соответствует оператору $\sqrt{A}$. Для определения последовательности в произвольном гильбертовом пространстве $\mathscr{H}$ используется изометрия пространств $\ell^{2}$ и $\mathscr{H}$.

В следующей теореме, обобщающей известный критерий Шура ([6; гл. 8, теорема 1], [7; с. 56]), показано, что бесселевы последовательности в пространстве $L^{2}(I)$ являются проекциями ортогональных систем из более широкого пространства $L^{2}(J)$.

ТеОрема 7. Пусть $I$ и $J$-открытые интервалы на числовой прямой $\mathbb{R}$, причем $I \subset J^{1}$. Функиии $\left\{f_{k}(x)\right\}_{k=1}^{\infty}$ образуют бесселеву последовательность в пространстве $L^{2}(I)$ с границей $B$ тогда и только тогда, когда в пространстве $L^{2}(J)$ существует ортогональная система $\left\{\varphi_{k}(x)\right\}_{k=1}^{\infty}$ такая, что

$$
\left\|\varphi_{k}(x)\right\|=\sqrt{B}, \quad k=1,2, \ldots, \quad u \quad f_{k}(x)=\varphi_{k}(x), \quad x \in I, k=1,2, \ldots .
$$

\footnotetext{
1 Допускаются и неограниченные интервалы. Знак $\subset$ применяется для обозначения строгого включения.
} 
ДокАЗАТЕЛЬСтво. Пусть функции $\mathscr{F}=\left\{f_{k}(x)\right\}_{k=1}^{\infty}$ образуют бесселеву последовательность в пространстве $L^{2}(I)$ с границей $B$. По лемме 3 имеем неравенство $B \mathbf{I}-G_{\mathscr{F}} \geqslant 0$. Следовательно (лемма 6$)$, в пространстве $L^{2}(J \backslash I)$ существует последовательность функций $\widetilde{\mathscr{F}}=\left\{\tilde{f}_{k}(x)\right\}_{k=1}^{\infty}$ такая, что

$$
G_{\widetilde{F}}=B I-G_{\mathscr{F}} .
$$

Определим функции $\Phi=\left\{\varphi_{k}(x)\right\}_{k=1}^{\infty}$ равенствами

$$
\varphi_{k}(x)=f_{k}(x)+\tilde{f}_{k}(x), \quad k=1,2, \ldots,
$$

и проверим их ортогональность:

$$
\left(\varphi_{k}, \varphi_{j}\right)=\int_{J} \varphi_{k}(x) \bar{\varphi}_{j}(x) d x=\int_{I} f_{k}(x) \bar{f}_{j}(x) d x+\int_{J \backslash I} \tilde{f}_{k}(x) \overline{\tilde{f}}_{j}(x) d x,
$$

следовательно,

$$
G_{\Phi}=G_{\mathscr{F}}+G_{\widetilde{F}}=G_{\mathscr{F}}+B I-G_{\mathscr{F}}=B I
$$

что и означает ортогональность и равенство всех норм.

Обратно, пусть в пространстве $L^{2}(J)$ задана ортогональная система $\left\{\varphi_{k}(x)\right\}_{k=1}^{\infty}$ такая, что $\left\|\varphi_{k}(x)\right\|=\sqrt{B}, k=1,2, \ldots$. Для каждого $k=1,2, \ldots$ определим функцию $f_{k}(x), x \in I$, как сужение функции $\varphi_{k}(x), x \in J$, на интервал $I$. Для произвольной последовательности $c=\left\{c_{k}\right\}_{k=1}^{\infty} \in \ell^{2}$ имеем

$$
\begin{aligned}
\left\|\sum_{k=1}^{\infty} c_{k} f_{k}\right\|_{L^{2}(I)}^{2} & =\int_{I}\left|\sum_{k=1}^{\infty} c_{k} f_{k}(x)\right|^{2} d x=\int\left|\sum_{J=1}^{\infty} c_{k} \varphi_{k}(x)\right|^{2} \cdot \chi_{I}(x) d x \\
& \leqslant \int_{J}\left|\sum_{k=1}^{\infty} c_{k} \varphi_{k}(x)\right|^{2} d x=\left\|\sum_{k=1}^{\infty} c_{k} \varphi_{k}\right\|_{L^{2}(J)}^{2} \leqslant B\|c\|_{\ell^{2}}^{2} .
\end{aligned}
$$

Таким образом, доказано (см. лемму 3$)$, что последовательность $\left\{f_{k}(x)\right\}_{k=1}^{\infty}$ бесселева.

Теорема 7 позволяет одним методом перенести известные теоремы о сходимости почти всюду ортогональных рядов на ряды по бесселевым последовательностям. Приведем три примера (теоремы Меньшова-Радемахера, Пэли-Зигмунда и Гарсиа).

ТЕорема 8 (аналог теоремы Меньшова-Радемахера для бесселевых систем). Пусть $I, J$ - открытье интерваль на $\mathbb{R} u I \subset J$. Пусть $\mathscr{F}=\left\{f_{k}\right\}_{k=1}^{\infty}, x \in I-$ произвольная бесселева последовательность на I с границей В. Если

$$
L:=\sum_{k=1}^{\infty}\left|c_{k}\right|^{2} \log _{2}^{2}(k+1)<\infty
$$

то ряд

$$
\sum_{k=1}^{\infty} c_{k} f_{k}(x)
$$


сходится почти всюду на I. Если

$$
S_{\mathscr{F}}^{*}(c, x):=\sup _{1 \leqslant N<\infty}\left|\sum_{k=1}^{N} c_{k} f_{k}(x)\right|, \quad c=\left\{c_{k}\right\}_{k=1}^{\infty},
$$

- мажоранта частных сумм ряда (2), то при условии (1) имеет место ощенка

$$
\left\|S_{\mathscr{F}}^{*}(c, x)\right\|_{L^{2}(I)} \leqslant C(B L)^{1 / 2},
$$

где $C$ - абсолютная постоянная.

ДокАЗАтЕЛЬство. По теореме 7 существует ортогональная система $\Phi=\left\{\varphi_{k}\right\}_{k=1}^{\infty}$ на $J,\left\|\varphi_{k}\right\|_{L^{2}(J)}=\sqrt{B}, k=1,2, \ldots$, такая, что $f_{k}(x)=\varphi_{k}(x), x \in I, k=1,2, \ldots$, где $B$ - бесселева граница $\mathscr{F}$. По теореме Меньшова-Радемахера [6; гл. 9, теорема 1] ряд

$$
\sum_{k=1}^{\infty} c_{k} \varphi_{k}(x)
$$

сходится почти всюду на $J$, причем существует абсолютная постоянная $C>0$ такая, что

$$
\left\|S_{\Phi}^{*}(c, x)\right\|_{L^{2}(J)} \leqslant C(B L)^{1 / 2} .
$$

Следовательно,

$$
\left\|S_{\mathscr{F}}^{*}(c, x)\right\|_{L^{2}(I)} \leqslant\left\|S_{\Phi}^{*}(c, x)\right\|_{L^{2}(J)} \leqslant C(B L)^{1 / 2} .
$$

Другой способ доказательства аналога теоремы Меньшова-Радемахера для бесселевых последовательностей основан на использовании факторизационной теоремы Морэ-Никишина по схеме Макарова [8; п. 2.3.3]. Ряд

$$
\sum_{k=1}^{\infty} c_{k} f_{k}(x)
$$

безусловно сходится в $L^{2}(I)$ для любого $c \in \ell^{2}$ (следствие 4). Отсюда следует [8; п. 2.3.3], что ряд

$$
\sum_{k=1}^{\infty} \frac{c_{k}}{\log _{2}(k+1)} f_{k}(x)
$$

сходится почти всюду на $I$.

Важным частным случаем бесселевой последовательности является фрейм или каркас.

ОПредЕЛЕНиЕ 9. Последовательность $\mathscr{F}=\left\{f_{k}\right\}_{k=1}^{\infty}$ элементов гильбертова пространства $\mathscr{H}$ называется фреймом для $\mathscr{H}$, если существуют постоянные $A>0$, $B>0$ такие, что

$$
A\|f\|^{2} \leqslant \sum_{k=1}^{\infty}\left|\left\langle f, f_{k}\right\rangle\right|^{2} \leqslant B\|f\|^{2}, \quad f \in \mathscr{H} .
$$


Числа $A$ и $B$ называются границами фрейма. Если возможен выбор границ $A=B$, то фрейм называется жестким. Заметим, что жесткие фреймы являются частным случаем ортоподобных систем, которые исследовались в работах Лукашенко и его учеников (см., например, [9], [10]).

Каждый фрейм является бесселевой системой. Известны примеры полных ${ }^{2}$ бесселевых систем, которые не являются фреймами [4; п. 5.4.6].

Фреймовым оператором $S=S_{\mathscr{F}}$ называется композиция операторов синтеза и анализа

$$
S: \mathscr{H} \rightarrow \mathscr{H}, \quad S f=D \circ C f=\sum_{k=1}^{\infty}\left\langle f, f_{k}\right\rangle f_{k} .
$$

Ряд, определяющий $S$, сходится безусловно для любого $f \in \mathscr{H}$ (следствие 4$)$. Оператор $S$ является ограниченным, обратимым, самосопряженным и положительным. Последовательность $\left\{S^{-1} f_{k}\right\}_{k=1}^{\infty}$ также образует фрейм для $\mathscr{H}$, который называют каноническим дуалъным фреймом для $\mathscr{F}$. Если $\mathscr{F}$ - фрейм с фреймовым оператором $S$, то

$$
f=\sum_{k=1}^{\infty}\left\langle f, S^{-1} f_{k}\right\rangle f_{k} \quad \forall f \in \mathscr{H} .
$$

Ряд (3) сходится безусловно в $\mathscr{H}$ для любого $f \in \mathscr{H}$ (следствие 4). Представление (3) называется фреймовым представлением элемента (сигнала) $f$. Перечисленные выше факты доказаны в [3] и [4; п. 5.1].

СлеДСтвиЕ 10. Если $\mathscr{F}$ - фрейм для пространства $L^{2}(I)$ u

$$
f \stackrel{L^{2}(I)}{=} \sum_{k=1}^{\infty}\left\langle f, S^{-1} f_{k}\right\rangle f_{k}
$$

- произвольное фреймовое представление, то ряд

$$
\sum_{k=1}^{\infty} \frac{\left\langle f, S^{-1} f_{k}\right\rangle}{\log _{2}(k+1)} f_{k}(x)
$$

сходится почти всюду на I.

ЛЕмма 11. Если $\left\{f_{k}\right\}_{k=1}^{\infty}-$ бесселева последовательность с границей $B$ в гильбертовом пространстве $\mathscr{H}$, то $\left\|f_{k}\right\|_{\mathscr{H}} \leqslant \sqrt{B}, k=1,2, \ldots$.

ДокАЗАтЕЛьСтво. Фиксируем натуральное число $m$ такое, что $\left\|f_{m}\right\|_{\mathscr{H}} \neq 0$. По определению бесселевой последовательности имеем

$$
\left|\left\langle f_{m}, f_{m}\right\rangle\right|^{2}+\sum_{k \neq m}\left|\left\langle f_{m}, f_{k}\right\rangle\right|^{2} \leqslant B\left\|f_{m}\right\|_{\mathscr{H}}^{2},
$$

или

$$
\left\|f_{m}\right\|_{\mathscr{H}}^{4} \leqslant\left\|f_{m}\right\|_{\mathscr{H}}^{4}+\sum_{k \neq m}\left|\left\langle f_{m}, f_{k}\right\rangle\right|^{2} \leqslant B\left\|f_{m}\right\|_{\mathscr{H}}^{2}
$$

отсюда следует, что $\left\|f_{m}\right\|_{\mathscr{H}} \leqslant \sqrt{B}$.

\footnotetext{
${ }^{2}$ Система $\mathscr{F}=\left\{f_{k}\right\}_{k=1}^{\infty}$ называется полной, если $\overline{\operatorname{span}}\left\{f_{k}\right\}=\mathscr{H}$. Очевидно, что фрейм является полной системой.
} 
Теорема 12 (аналог теоремы Пэли-Зигмунда для бесселевых систем). Пусть $J$ - открытый интервал на $\mathbb{R},\left\{f_{k}(x)\right\}_{k=1}^{\infty}$ - бесселева последовательность в $L^{2}(J)$. Если $c=\left\{c_{k}\right\}_{k=1}^{\infty} \in \ell^{2}$, то при почти всех $t \in[0,1]$ ряд

$$
\sum_{k=1}^{\infty} r_{k}(t) c_{k} f_{k}(x), \quad x \in J
$$

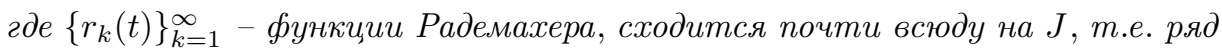

$$
\sum_{k=1}^{\infty} \pm c_{k} f_{k}(x), \quad x \in J
$$

сходится почти всюду на $J$ при почти всех выборах знаков у членов ряда.

ДокАЗАТЕЛЬСтво. Применяя лемму 11, имеем

$$
\int_{J} \sum_{k=1}^{n}\left|c_{k}\right|^{2}\left|f_{k}(x)\right|^{2} d x \leqslant B \cdot \sum_{k=1}^{n}\left|c_{k}\right|^{2} \leqslant B \cdot \sum_{k=1}^{\infty}\left|c_{k}\right|^{2} .
$$

Отсюда по теореме Б. Леви следует, что для почти всех $x \in J$ конечна сумма ряда

$$
\sum_{k=1}^{\infty}\left|c_{k}\right|^{2}\left|f_{k}(x)\right|^{2}
$$

Следовательно [6; гл. 2, теорема 11], ряд

$$
\sum_{k=1}^{\infty} r_{k}(t) c_{k} f_{k}(x)
$$

сходится почти всюду на $J$ при почти каждом $t \in[0,1]$.

Альтернативный способ доказательства основан на теореме 7. Если $\left\{f_{k}(x)\right\}_{k=1}^{\infty}-$ бесселева последовательность в $L^{2}(I)$, то $f_{k}(x)=\varphi_{k}(x), x \in I, k=1,2, \ldots$, для некоторой ортогональной системы

$$
\left\{\varphi_{k}(x)\right\}_{k=1}^{\infty}, \quad\left\|\varphi_{k}\right\|_{L^{2}(J)}=\sqrt{B}, \quad k=1,2, \ldots,
$$

из пространства $L^{2}(J)$, где $I \subset J$. Для ряда $\sum_{k=1}^{\infty} c_{k} \varphi_{k}(x)$ можно применить классический результат об ортогональных рядах [6; с. 66] и "спроектировать" его на ряд $\sum_{k=1}^{\infty} c_{k} f_{k}(x)$.

Теорема 13 (аналог теоремы Гарсиа для бесселевых систем). Пусть $I, J$ - omкрытые интервалы на $\mathbb{R} u I \subset J$. Пусть $\mathscr{F}=\left\{f_{k}(x)\right\}_{k=1}^{\infty}, x \in I,-$ произвольная бесселева последовательность на I с гранищей $B$. Для любого ряда

$$
\sum_{k=1}^{\infty} c_{k} f_{k}(x), \quad c=\left\{c_{k}\right\}_{k=1}^{\infty} \in \ell^{2}
$$

существует перестановка натурального ряда $\sigma=\{\sigma(k)\}_{k=1}^{\infty}$ такая, что ряд

$$
\sum_{k=1}^{\infty} c_{\sigma(k)} f_{\sigma(k)}(x)
$$


сходится почти всюду на I и, кроме того, существует абсолютная постоянная $C>0$ такая, что

$$
\int_{I \leqslant N<\infty}\left|\sum_{k=1}^{N} c_{\sigma(k)} f_{\sigma(k)}(x)\right|^{2} d x \leqslant C \cdot\|c\|_{\ell^{2}}^{2} .
$$

ДоказАтЕЛЬСтво. По теореме 7 существует ортогональная система $\Phi=\left\{\varphi_{k}\right\}_{k=1}^{\infty}$ на $J,\left\|\varphi_{k}\right\|_{L^{2}(J)}=\sqrt{B}, k=1,2, \ldots$, такая, что $f_{k}(x)=\varphi_{k}(x), x \in I, k=1,2, \ldots$, где $B$ - бесселева граница $\mathscr{F}$. Для ряда $\sum_{k=1}^{\infty} c_{k} \varphi_{k}(x)$ справедлива теорема Гарсиа [6; гл. 2, теорема 16]. В [10] показано, что теорема Гарсиа сохраняет силу и для комплексных чисел $\left\{c_{k}\right\}_{k=1}^{\infty}$. Таким образом, существует перестановка натурального ряда $\sigma=\{\sigma(k)\}_{k=1}^{\infty}$ такая, что ряд

$$
\sum_{k=1}^{\infty} c_{\sigma(k)} \varphi_{\sigma(k)}(x)
$$

сходится почти всюду на $J$ и имеет место неравенство

$$
\int_{J} \sup _{1 \leqslant N<\infty}\left|\sum_{k=1}^{N} c_{\sigma(k)} \varphi_{\sigma(k)}(x)\right|^{2} d x \leqslant C \cdot\|c\|_{\ell^{2}}^{2}
$$

с абсолютной постоянной $C$.

Отсюда следует сходимость почти всюду на $I$ ряда

$$
\sum_{k=1}^{\infty} c_{\sigma(k)} f_{\sigma(k)}(x)
$$

и неравенство

$$
\int_{I} \sup _{1 \leqslant N<\infty}\left|\sum_{k=1}^{N} c_{\sigma(k)} \varphi_{\sigma(k)}(x)\right|^{2} d x \leqslant \int_{J} \sup _{1 \leqslant N<\infty}\left|\sum_{k=1}^{N} c_{\sigma(k)} \varphi_{\sigma(k)}(x)\right|^{2} d x \leqslant C \cdot\|c\|_{\ell^{2}}^{2} .
$$

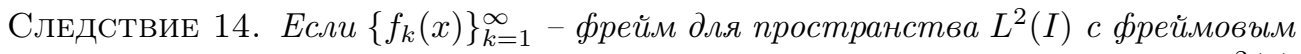
оператором $S, I \subset J$ - открытые интерваль на $\mathbb{R}$, то для любого $f \in L^{2}(I)$ существует перестановка натурального ряда $\sigma=\{\sigma(k)\}_{k=1}^{\infty}$ такая, что ряд

$$
\sum_{k=1}^{\infty}\left\langle f, S^{-1} f_{\sigma(k)}\right\rangle f_{\sigma(k)}(x)=f(x)
$$

почти всюду на $I$.

ДокАЗАТЕЛЬСтво. Каждый фрейм является бесселевой системой, фреймовы коэффициенты $\left\{f, S^{-1} f_{k}\right\}_{k=1}^{\infty} \in \ell^{2}$, сходимость фреймова представления является безусловной в $L^{2}(I)$. Эти три факта и аналог теоремы Гарсиа для бесселевых последовательностей доказывают следствие.

Следующая теорема показывает, какие бесселевы последовательности допускают продолжение до полной ортогональной системы. Такими последовательностями являются жесткие фреймы с двумя дополнительными условиями на матрицу Грама. Теорема обобщает известную теорему Олевского [6; гл. 8, теорема 3], [7; с. 57]. 
Теорема 15. Пусть $I$ и $J$-открытые интервалы на числовой прямой $\mathbb{R}$, причем $I \subset J$, и пусть $B>0$. Функиии $\mathscr{F}=\left\{f_{k}(x)\right\}_{k=1}^{\infty} \subset L^{2}(I)$ могут быть продолжены до элементов полной ортогональной системы

$$
\Phi=\left\{\varphi_{k}(x)\right\}_{k=1}^{\infty} \subset L^{2}(J), \quad\left\|\varphi_{k}(x)\right\|=\sqrt{B}, \quad k=1,2, \ldots,
$$

тогда и только тогда, когда выполнены следующие условия:

1) последовательность Я્F образует жесткий фрейм пространства $L^{2}(I)$ с границей $B$;

2) $B G_{\mathscr{F}}=G_{\mathscr{F}}^{2}$;

3) матрица $B \mathbf{I}-G_{\mathscr{F}}$ имеет бесконечный ранг.

ДокАЗАТЕЛьСтво. Пусть последовательность $\Phi=\left\{\varphi_{k}(x)\right\}_{k=1}^{\infty}$ образует полную ортогональную систему в пространстве $L^{2}(J),\left\|\varphi_{k}(x)\right\|=\sqrt{B}, k=1,2, \ldots$, и пусть

$$
f_{k}(x)=\varphi_{k}(x) \chi_{I}(x), \quad x \in J .
$$

Проверим выполнение условий 1$)-3)$. Функцию $f \in L^{2}(I)$ продолжаем нулем на $J \backslash I$ и такое продолжение вновь обозначаем $f$. В силу полноты системы $\Phi$ имеем

$$
\sum_{k=1}^{\infty}\left|\left(f, f_{k}\right)\right|^{2}=B \sum_{k=1}^{\infty}\left|\int_{J} f \chi_{I} \frac{\bar{\varphi}_{k}}{\sqrt{B}} d x\right|^{2}=B\left\|f \chi_{I}\right\|_{L^{2}(J)}^{2}=B\|f\|_{L^{2}(I)}^{2},
$$

таким образом, выполнено условие 1$)$.

Определим систему функций

$$
\widetilde{\Phi}=\left\{\tilde{\varphi}_{k}(x)\right\}_{k=1}^{\infty},
$$

полагая

$$
\tilde{\varphi}_{k}(x)=\varphi_{k}(x) \chi_{J \backslash I}=\varphi_{k}(x)-f_{k}(x) .
$$

Заметим, что

$$
G_{\widetilde{\Phi}}=B \mathbf{I}-G_{\mathscr{F}} .
$$

С другой стороны, в силу равенства Парсеваля имеем

$$
\left(G_{\widetilde{\Phi}}\right)_{j, k}=\int_{J} \tilde{\varphi}_{k} \overline{\tilde{\varphi}}_{j} d x=\sum_{n=1}^{\infty}\left\langle\tilde{\varphi}_{k}, \frac{\varphi_{n}}{\sqrt{B}}\right\rangle\left\langle\frac{\varphi_{n}}{\sqrt{B}}, \tilde{\varphi}_{j}\right\rangle=\frac{1}{B} \sum_{n=1}^{\infty}\left(G_{\widetilde{\Phi}}\right)_{j, n}\left(G_{\widetilde{\Phi}}\right)_{n, k} .
$$

В матричной форме последнее соотношение имеет вид

$$
G_{\widetilde{\Phi}}=\frac{1}{B} G_{\widetilde{\Phi}}^{2},
$$

или

$$
B G_{\mathscr{F}}=G_{\mathscr{F}}^{2} .
$$

Получаем условие 2).

Полнота системы $\widetilde{\Phi}$ в пространстве $L^{2}(J \backslash I)$ влечет условие 3$)$.

Рассмотрим систему функций $\mathscr{F}$, для которой выполнены условия 1$)-3)$. Обозначим через $\tilde{g}_{j, k}(j, k)$-й элемент матрицы $B \mathbf{I}-G_{\mathscr{F}}$. Пусть $\left\{\psi_{k}\right\}_{k=1}^{\infty}-$ произвольная полная ортонормированная система в пространстве $L^{2}(J \backslash I)$. Определим функции

$$
p_{j}(x)=\sum_{k=1}^{\infty} \tilde{g}_{j, k} \psi_{k}(x), \quad j=1,2, \ldots
$$


Имеем, во-первых,

$$
\left\langle p_{j}, p_{m}\right\rangle=\sum_{k=1}^{\infty} \tilde{g}_{j, k} \overline{\tilde{g}}_{m, k}=\sum_{k=1}^{\infty} \tilde{g}_{j, k} \tilde{g}_{k, m}=B \tilde{g}_{j, m},
$$

по условию 2).

Во-вторых, в силу 3) существует изометрия $U$ между $\overline{\operatorname{span}}\left\{p_{j}\right\}$ и $L^{2}(J \backslash I)$, причем $\left\langle U p_{j}, U p_{m}\right\rangle=B \tilde{g}_{j, m}$.

Теперь мы определяем полную ортонормированную систему $\Phi$, которая является продолжением системы $\mathscr{F}$ :

$$
\Phi=\left\{\varphi_{k}\right\}, \quad \varphi_{k}(x)= \begin{cases}f_{k}(x), & x \in I, \\ \frac{1}{\sqrt{B}}\left(U p_{k}\right)(x), & x \in J \backslash I .\end{cases}
$$

Непосредственно из определения получаем равенство для матриц Грама

$$
G_{\Phi}=G_{\mathscr{F}}+B \mathbf{I}-G_{\mathscr{F}}=B \mathbf{I}
$$

которое показывает, что система $\Phi$ ортонормирована и $\left\|\varphi_{k}\right\|=\sqrt{B}, k=1,2, \ldots$.

Для доказательства полноты проверяем, что система $\Phi$ является жестким фреймом с границей $B$ для пространства $L^{2}(J)$. Определение жесткого фрейма достаточно проверять для элементов некоторой полной системы пространства $L^{2}(J)$. В качестве такой “тестовой системы” определяем систему

$$
\Theta=\left\{\theta_{m}, \tilde{\theta}_{m}\right\}=\left\{\varphi_{m} \chi_{I}, \varphi_{m} \chi_{J \backslash I}\right\} .
$$

Полнота системы $\Theta$ непосредственно следует из построения,

$$
\begin{aligned}
\sum_{k=1}^{\infty}\left|\left\langle\theta_{m}, \varphi_{k}\right\rangle\right|^{2} & =\sum_{k=1}^{\infty}\left\langle f_{m}, f_{k}\right\rangle\left\langle f_{k}, f_{m}\right\rangle=\sum_{k=1}^{\infty} g_{m, k} g_{k, m} \\
& =\left(G_{\mathscr{F}}\right)_{m, m}^{2}=\left(B G_{\mathscr{F}}\right)_{m, m}=B\left\|\theta_{m}\right\|^{2}, \\
\sum_{k=1}^{\infty}\left|\left\langle\tilde{\theta}_{m}, \varphi_{k}\right\rangle\right|^{2} & =\sum_{k=1}^{\infty}\left\langle\frac{1}{\sqrt{B}} U p_{m}, \varphi_{k} \chi_{J \backslash I}\right\rangle\left\langle\varphi_{k} \chi_{J \backslash I}, U p_{m}\right\rangle \\
& =\frac{1}{B} \sum_{k=1}^{\infty}\left\langle p_{m}, p_{k}\right\rangle\left\langle p_{k}, p_{m}\right\rangle=\frac{1}{B} \sum_{k=1}^{\infty} B \tilde{g}_{m, k} B \tilde{g}_{k, m} \\
& =B\left(B \mathbf{I}-G_{\mathscr{F}}\right)_{m, m}=B\left\|\tilde{\theta}_{m}\right\|^{2} .
\end{aligned}
$$

Таким образом показано, что система $\Phi$ является жестким фреймом, следовательно, полной системой в пространстве $L^{2}(I)$.

Примеры. 1) Пусть $\left\{e_{k}\right\}_{k=1}^{\infty}-$ орты пространства $\ell^{2}$,

$$
\left\{f_{k}\right\}_{k=1}^{\infty}=\left\{e_{1}, \frac{1}{\sqrt{2}} e_{2}, \frac{1}{\sqrt{2}} e_{2}, \frac{1}{\sqrt{3}} e_{3}, \frac{1}{\sqrt{3}} e_{3}, \frac{1}{\sqrt{3}} e_{3}, \ldots\right\},
$$

т.е. в последовательности $\left\{f_{k}\right\}_{k=1}^{\infty}$ вектор $e_{k} / \sqrt{k}$ повторяется $k$ раз. 
Для каждого $f \in \ell^{2}$

$$
\sum_{k=1}^{\infty}\left|\left\langle f, f_{k}\right\rangle\right|^{2}=\sum_{k=1}^{\infty} k\left|\left\langle f, \frac{1}{\sqrt{k}}\right\rangle\right|^{2}=\|f\|^{2} .
$$

Получаем, что последовательность $\left\{f_{k}\right\}_{k=1}^{\infty}$ образует жесткий фрейм для пространства $\ell^{2}$ с границей $A=B=1$. Непосредственно проверяется, что для матрицы Грама последовательности $\mathscr{F}=\left\{f_{k}\right\}_{k=1}^{\infty}$ имеем $G_{\mathscr{F}}^{2} \neq G_{\mathscr{F}}$, и соответствующая $\mathscr{F}$ последовательность в пространстве $L^{2}(I)$ не может быть продолжена до полной ортонормированной системы в $L^{2}(J)$.

2) Если $\Phi=\left\{\varphi_{k}\right\}_{k=1}^{\infty}-$ полная ортонормированная система в $L^{2}(J)$, то система $\left\{\varphi_{k} \chi_{I}\right\}_{k=1}^{\infty}$ доставляет пример жесткого фрейма для пространства $L^{2}(I)$, который не является базисом. Функция $f \in L^{2}(I)$ может быть разными способами продолжена на интервал $J$. Каждому продолжению соответствует единственный набор коэффициентов представления по системе $\Phi$. Любой из таких наборов в пространстве $L^{2}(I)$ представляет функцию $f$ по системе $\left\{\varphi_{k} \chi_{I}\right\}_{k=1}^{\infty}$. Таким образом, система $\left\{\varphi_{k} \chi_{I}\right\}_{k=1}^{\infty}$ не является базисом.

\section{СПИСОК ЦИТИРОВАННОЙ ЛИТЕРАТУРЫ}

[1] И. Добеши, Десять лекиий по вейвлетам, НИЦ "Регуляр. и хаотич. динамика", М.-Ижевск, 2001.

[2] Б. С. Кашин, Т. Ю. Куликова, “Замечание об описании фреймов общего вида”, Maтем. заметки, 72:6 (2002), 941-945.

[3] С. Малла, Вэйвлеты в обработке сигналов, Мир, М., 2005.

[4] O. Christensen, An Introduction to Frames and Riesz Bases, Birkhäuser, Boston, 2003.

[5] У. Рудин, Функционалъный анализ, Мир, М., 1975.

[6] Б. С. Кашин, А. А. Саакян, Ортогональные ряды, АФЦ, М., 1999.

[7] A.M. Olevskii, Fourier Series with Respect to General Orthogonal Systems, Springer-Verlag, Berlin-Heidelberg-New York, 1975.

[8] Б. М. Макаров, " $p$-абсолютно суммирующие операторы и некоторые их приложения", Алгебра и анализ, 3:2 (1991), 1-76.

[9] Т. П. Лукашенко, "О коэффициентах систем разложения, подобных ортогональным", Матем. сборник, 188:12 (1997), 57-72.

[10] Т. П. Лукашенко, Т. В. Родионов, "О сходимости рядов по обобщенным ортоподобным системам", Фундам. и прикл. матем., 6:3 (2000), 813-829.

\section{С. Я. Новиков}

Самарский государственный университет

E-mail: nvks@ssu.samara.ru
Поступило

20.03.2006

Исправленный вариант 26.09.2006 